\title{
Presencia de factores de riesgo asociados a la diseminación de brucelosis al humano en unidades procesadoras de leche y mataderos de la provincia Manabí, Ecuador
}

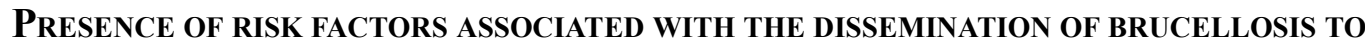 \\ HUMANS IN MILK PROCESSING AND SLAUGHTERING UNITS OF THE MANABí PROVINCE, \\ ECUADOR
}

\author{
Marina Dalia Zambrano Aguayo ${ }^{1,3}$, Isabel Victoria Díaz Salavarria ${ }^{1}$, \\ Miguel Pérez-Ruano ${ }^{2}$
}

\section{Resumen}

\begin{abstract}
Se realizó una investigación epidemiológica transversal para determinar los factores de riesgo asociados a la diseminación de brucelosis a los humanos en centro procesadores de leche y mataderos de siete cantones de la provincia Manabí, Ecuador, entre septiembre y noviembre de 2015. Se realizó un censo para determinar las unidades que elaboraban subproductos lácteos y los mataderos. Se seleccionaron 119 lecherías dedicadas a la elaboración artesanal de productos lácteos, siete mataderos y siete centros procesadores de productos lácteos, y se caracterizaron en cuanto a los tipos de productos que elaboran y la presencia de factores de riesgo como causales de brucelosis en humanos. Se determinó la presencia de anticuerpos contra Brucella spp en la leche utilizada como materia prima mediante la prueba del anillo. El 97.6\% de las unidades elaboran un solo producto (queso artesanal). Se encontró la presencia de $85.0 \%$ de ocurrencia de factores de riesgo en las lecherías que procesan leche, valor superior al encontrados en las otras unidades $(\mathrm{p}<0.05)$ Los principales factores de riesgos detectados relacionados con las instalaciones fueron: presencia de otras especies de animales $(100 \%)$, no tratamiento de residuales $(85.7 \%)$, flujo de trabajo inadecuado $(85.7 \%)$ y no control de la materia prima (57.1\%). En los factores relacionados con el personal en los tres tipos de unidades destacan el consumo de alimentos riesgosos en todas las unidades y la no investigación serológica periódica del personal en más del $80 \%$ de las unidades. Se comprobó el uso de leche positiva a la prueba del anillo en la elaboración de productos lácteos, especialmente en los centros procesadores de leche.
\end{abstract}

Palabras clave: brucelosis; factores de riesgo; leche; Ecuador

\footnotetext{
${ }^{1}$ Departamento de Medicina Veterinaria, Facultad de Ciencias Veterinarias, Universidad Técnica de Manabi (UTM), Portoviejo, Ecuador

${ }^{2}$ Departamento de Medicina Preventiva, Facultad de Medicina Veterinaria, Universidad Agraria de la Habana (UNAH), Mayabeque, Cuba

${ }^{3}$ Email:mazambrano@utm.edu.ec
}

Recibido: 30 de marzo de 2017

Aceptado para publicación: 13 de agosto de 2017 
A cross-sectional epidemiological investigation was carried out to determine the risk factors associated with the dissemination of brucellosis to humans in milk processing centres and slaughterhouses in seven cantons of the province of Manabí, Ecuador, between September and November 2015. A census was conducted to quantify farms processing milk, milk processing centres and slaughterhouses. A total of 119 dairy farms, seven slaughterhouses and seven milk processing centres were selected. They were characterized in terms of the types of products produced and on the presence of risk factors as causes of brucellosis in humans. The presence of antibodies against Brucella spp was determined in raw milk by the milk ring test. Results showed that $97.6 \%$ of the units make a single product (artisanal cheese). The presence of $85.0 \%$ of risk factor was found in milk processing dairies, value higher than that found in the other units $(\mathrm{p}<0.05)$. The main risk factors detected related to the facilities were: the presence of other species of animals $(100 \%)$, no treatment of residuals $(85.7 \%)$, inadequate workflow $(85.7 \%)$ and no control of the raw milk (57.1\%). The factors related to personnel in the three types of units include the consumption of hazardous foods in all units and the non-periodical serological checks of staff in more than $80 \%$ of the units. The use of positive milk to the ring test in the elaboration of milk products was observed, especially in milk processing centres.

Key words: brucellosis, risk factors, milk, Ecuador

\section{INTRODUCCIÓN}

La brucelosis es una enfermedad infecciosa reemergente de distribución mundial de gran importancia (Aréstegui et al., 2001; Bhanu et al., 2013; Zhong et al., 2013) y que tiene repercusiones negativas sobre la economía de los países y la salud humana; en particular, los ganaderos y, entre estos, los que realizan faenado de los animales (Agrocalidad, 2009).

La incidencia de la enfermedad en los humanos varía entre regiones y países (Dean et al., 2012). Su prevalencia está asociada a las condiciones socioeconómicas de cada país, región o localidad. En países en vías de desarrollo, donde se utiliza un sistema tradicional de manejo de los animales y los sistemas sanitarios son deficientes o inexistentes, afecta a la población en general (Paredes-Vargas, 2012).
La infección tiene carácter ocupacional para los veterinarios, trabajadores de mataderos y ganaderos que manejan animales infectados y canales, así como fetos abortados y placentas (OIE, 2016). La transmisión puede ocurrir a través del ambiente contaminado, por contacto directo con animales infectados (Ron-Román et al., 2014), por ingesta de productos lácteos elaborados con leche sin pasteurizar o por el consumo de alimentos contaminados (Osman et al., 2015). La transmisión de la enfermedad se puede favorecer ante deficiencias en bioseguridad y en higiene (García-Juárez et al., 2014). Además, existe un riesgo laboral a nivel de laboratorio, a partir de aerosoles al manipular antígenos (Luigi et al., 2006).

Organismos internacionales como la Organización Mundial de la Salud (OMS), la Organización Mundial de Sanidad Animal (OIE) y la Organización Panamericana de la Salud (OPS) exhortan a los países a buscar 
estrategias que disminuyan las probabilidades de riesgo donde la enfermedad todavía existe (OPS/OMS, 2005; OIE, 2016). La pasteurización de la leche ha minimizado el número de brotes humanos; sin embargo, la brucelosis es considerada como una enfermedad reemergente en ciertas regiones del mundo (Motarjemi et al., 2014).

En el caso particular de Ecuador y, en específico de la provincia Manabí, su población rural se encuentra en permanente riesgo debido a la cercanía con la fuente de infección (D'Anastasio et al., 2011). En un estudio previo realizado en la región (Zambrano y Pérez, 2015), se reportó una seroprevalencia de brucelosis bovina de $2.33 \%$ y una seroprevalencia de brucelosis de $1 \%$ en los trabajadores de los hatos ganaderos y de los mataderos investigados; sin embargo, se conoce muy poco sobre los factores de riesgos que se asocian a la diseminación de brucelosis a humanos en fincas lecheras, centros procesadores de leche y en mataderos; lo cual dificulta la confección de estrategias para la prevención y el control de la enfermedad. Mediante este estudio se tuvo como objetivo determinar la presencia de factores de riesgo asociados a la diseminación de brucelosis en centros y fincas procesadores de leche, así como en mataderos de la provincia Manabí.

\section{Materiales y Métodos}

Se realizó un estudio epidemiológico transversal entre septiembre y noviembre de 2015 en los cantones Bolívar, El Carmen, Chone, Jama, Junín, Sucre y Tosagua de la provincia de Manabí. En forma previa, se realizó un censo para determinar el total de unidades industriales y artesanales que procesan leche y los mataderos existentes en los cantones en estudio, entrevistándose a las autoridades correspondientes del Ministerio de Agricultura, Ganadería, Acuacultura y Pesca (MAGAP), consejos cantonales y provinciales, $\mathrm{y}$ asociaciones ganaderas.
Cuadro 1. Distribución por cantones de lecherías procesadoras de leche, centros de procesamiento de leche y de mataderos en la provincia de Manabí, Ecuador

\begin{tabular}{lccc}
\hline Cantón & Lecherías $^{1}$ & CdeP $^{2}$ & Mataderos \\
\hline Bolívar & 16 & 0 & 1 \\
Chone & 25 & 5 & 1 \\
El Carmen & 20 & 1 & 1 \\
Jama & 14 & 0 & 1 \\
Junín & 17 & 0 & 1 \\
Sucre & 13 & 1 & 1 \\
Tosagua & 14 & 0 & 1 \\
\hline Total & 119 & 7 & 7 \\
${ }^{1}$ Lecherías procesadoras de leche \\
${ }^{2}$ Centros de procesamiento de leche
\end{tabular}

A partir del censo realizado, se seleccionó el $10 \%$ de las lecherías procesadoras de leche (119 unidades), el 100\% de los mataderos ( 7 unidades) y el $100 \%$ de los centros de procesamiento de leche de forma artesanal, industrial y semi-industrial (7 unidades) (Cuadro 1). En las unidades seleccionadas se realizó una encuesta epidemiológica sobre sus instalaciones y procedimientos, y se recolectó información sobre los productos que elaboran y la presencia de factores de riesgo para la afectación de los humanos. Además, en los centros y fincas procesadores de leche se recolectaron muestras de leche para determinar la presencia de anticuerpos contra Brucella spp.

Se consideraron los siguientes factores de riesgo en relación a las instalaciones: presencia de otras especies animales, no se realiza la prueba de anillo a la materia prima, no pasteurizan la leche antes de elaborar los productos, mala higiene de la instalación, mala higiene de los equipos, mala higiene de los utensilios, no se realiza desinfección de la instalación, no hay tratamiento de residuos, hay presencia de vectores, no hay filtro sanitario, 
condiciones inadecuadas de las instalaciones, flujo de trabajo incorrecto, instrumental inadecuado, no existe aislamiento externo, y no hay control de la materia prima.

En el caso del personal se evaluaron los siguientes factores de riesgo:

- Consumo de alimentos riesgosos de la unidad. En el caso de las fincas procesadoras de leche y los centros de procesamiento de leche sin pasteurizar se consideró como productos riesgosos la leche y productos lácteos sin pasteurizar. En el caso de los mataderos se consideró la carne sin procesar, así como fetos, vísceras, etc.

- Presencia de malos hábitos higiénicos

- El no uso de ropa sanitaria

- No uso de otros medios de protección (delantales, guantes, mallas protectoras de heridas, etc.)

- Historial de enfermedad por brucelosis

- Historial de enfermedad por brucelosis de los familiares

- No investigación de brucelosis en las personas

El porcentaje de factores de riesgo presentes (FRP) en cada unidad fue calculada con la fórmula siguiente: (Factores presentes/total de factores evaluados)*100. Asimismo, el porcentaje de factores presentes en lecherías procesadoras de leche, centros de procesamiento de leche y mataderos fue calculada con la fórmula siguiente: suma de los factores presentes en todas las lecherías o centros o mataderos dividido entre la suma de los factores evaluados en todas las lecherías o centros o mataderos y multiplicado por 100 .

Para determinar la presencia de anticuerpos contra Brucella spp en cada unidad se tomaron $20 \mathrm{ml}$ de leche cruda antes de la elaboración de los productos y se investigó mediante la prueba de anillo de la leche, según lo descrito por la OIE (2016), utilizando un antígeno comercial (LABIOFAM, Cuba).
Con la información registrada se hicieron análisis de comparación de proporciones para determinar diferencias en porcentaje de FRP entre los tipos de unidades estudiadas, así como para determinar posibles diferencias significativas en la presencia de anticuerpos contra Brucella spp entre centros y fincas procesadores de leche.

\section{Resultados}

El 94.4\% de las unidades procesadores de leche se dedican a la elaboración de un solo tipo de producto lácteo, básicamente el queso fresco (Cuadro 2).

En los cuadros 3 y 4 se puede observar el elevado porcentaje de FRP en los tres tipos de unidades en estudio, tanto en relación con las instalaciones como con el personal. Las mayores deficiencias se presentaron en las lecherías procesadoras de leche con el $85.0 \%$ de factores de riesgo relacionados con las instalaciones y con $67.7 \%$ de factores de riesgo relacionados con el personal.

Cuadro 2. Tipos de productos lácteos que elaboran las lecherías y centros procesadores de leche de la provincia de Manabí, Ecuador

\begin{tabular}{lcc}
\hline Productos & Unidades & $\%$ \\
\hline Queso industrial & 3 & 2.4 \\
Queso artesanal & 123 & 97.6 \\
Yogurt artesanal & 3 & 2.4 \\
Yogurt industrial & 2 & 1.6 \\
Mantequilla artesanal & 3 & 2.4 \\
Mantequilla industrial & 1 & 0.8 \\
Requesón industrial & 4 & 3.2 \\
Requesón artesanal & 3 & 2.4 \\
Dulce de leche artesanal & 1 & 0.8 \\
Dulce de leche industrial & 1 & 0.8 \\
\hline
\end{tabular}


Cuadro 3. Presencia de factores de riesgo como causal de brucelosis en humanos relacionados con las instalaciones las lecherías (L) y centros de procesamiento (CdeP) de leche y de mataderos de la provincia de Manabí, Ecuador

\begin{tabular}{|c|c|c|c|c|c|c|}
\hline \multirow{2}{*}{ Variable evaluada } & \multicolumn{2}{|c|}{$\begin{array}{c}\mathrm{L} \\
(\mathrm{n}=7)\end{array}$} & \multicolumn{2}{|c|}{$\begin{array}{l}\text { CdeP } \\
(n=119)\end{array}$} & \multicolumn{2}{|c|}{$\begin{array}{l}\text { Matadero } \\
\quad(\mathrm{n}=7)\end{array}$} \\
\hline & $\mathrm{n}$ & $\%$ & $\mathrm{n}$ & $\%$ & $\mathrm{n}$ & $\%$ \\
\hline $\begin{array}{l}\text { Presencia de otras especies de } \\
\text { animales }\end{array}$ & 0 & 0 & 77 & 64.7 & 7 & 100.0 \\
\hline $\begin{array}{l}\text { No se realiza prueba de anillo a la } \\
\text { materia prima }\end{array}$ & 0 & 0 & 119 & 100.0 & & \\
\hline $\begin{array}{l}\text { No pasteurizan la leche antes de } \\
\text { elaborar los productos }\end{array}$ & 3 & 42.9 & 119 & 100.0 & & \\
\hline Mala higiene de la instalación & 0 & 0 & 98 & 82.4 & 6 & 85.7 \\
\hline Mala higiene de los equipos & 1 & 14.3 & & & 3 & 42.9 \\
\hline Mala higiene de los utensilios & 1 & 14.3 & 96 & 80.7 & 3 & 42.9 \\
\hline $\begin{array}{l}\text { No se realiza desinfección de la } \\
\text { instalación }\end{array}$ & 0 & 0 & 71 & 59.7 & 0 & 0 \\
\hline No hay tratamiento de residuales & 6 & 85.7 & 119 & 100.0 & 7 & 100.0 \\
\hline Hay presencia de vectores & 2 & 28.6 & 104 & 87.4 & 2 & 28.6 \\
\hline No hay filtro sanitario & 2 & 28.6 & 119 & 100.0 & 7 & 100.0 \\
\hline $\begin{array}{l}\text { Condiciones inadecuadas de las } \\
\text { instalaciones }\end{array}$ & 3 & 42.9 & 102 & 85.7 & 5 & 71.4 \\
\hline Flujo de trabajo inadecuado & 6 & 85.7 & 76 & 63.9 & 4 & 57.1 \\
\hline Instrumental inadecuado & 2 & 28.6 & 110 & 92.4 & 3 & 42.9 \\
\hline No existe aislamiento externo & 1 & 14.3 & 87 & 73.1 & 2 & 28.6 \\
\hline No hay control de la materia prima & 4 & 57.1 & 119 & 100.0 & 0 & 0 \\
\hline Promedio & & 29.5 & & 85.0 & & 53.9 \\
\hline
\end{tabular}

En el caso de factores relacionados con las instalaciones (Cuadro 3), en los centros de procesamiento de leche se destacan la falta de tratamiento de los residuales y el inadecuado flujo de trabajo con más del $80 \%$ de ocurrencia. En el caso de las lecherías procesadoras de leche, la mayoría de factores de riesgo se encuentran presentes, pero destacan la no realización de la prueba del anillo de la leche, la no pasteurización de la leche, la falta de tratamiento de los residuales, la ausencia de filtro sanitario y el no control de la materia prima $(100 \%$ en todos los ca- sos). En el caso de los mataderos, destacan la presencia de otras especies animales, la falta de tratamiento de residuales y la falta de filtro sanitario ( $100 \%$ en todos los casos).

En los factores relacionados con el personal (Cuadro 4), en los tres tipos de unidades destaca la ocurrencia de consumo de alimentos riesgosos $(100 \%)$ y la no investigación serológica periódica del personal (85.7$100 \%$ ). Además, se observó la no utilización de ropa sanitaria ni medios de protección en las lecherías procesadoras de leche $(100 \%)$. 
Cuadro 4. Presencia de factores de riesgo asociados al personal como causal de brucelosis en humanos en lecherías (L) y centros de procesamiento (CdeP) de leche y de mataderos de la provincia de Manabí, Ecuador

\begin{tabular}{lcccccc}
\hline \multirow{2}{*}{ Variable evaluada } & \multicolumn{2}{c}{$\begin{array}{c}\text { CdeP } \\
(\mathrm{n}=7)\end{array}$} & \multicolumn{2}{c}{$\begin{array}{c}\mathrm{L} \\
(\mathrm{n}=119)\end{array}$} & \multicolumn{2}{c}{$\begin{array}{c}\text { Matadero } \\
(\mathrm{n}=7)\end{array}$} \\
\cline { 2 - 8 } & $\mathrm{n}$ & $\%$ & $\mathrm{n}$ & $\%$ & $\mathrm{n}$ & $\%$ \\
\hline $\begin{array}{l}\text { Consume alimentos riesgosos en la } \\
\text { unidad }\end{array}$ & 7 & 100.0 & 119 & 100.0 & 7 & 100.0 \\
$\begin{array}{l}\text { Malos hábitos higiénicos } \\
\text { No uso de ropa sanitaria }\end{array}$ & 0 & 0 & 89 & 74.8 & 0 & 0 \\
$\begin{array}{l}\text { No uso de otros medios de } \\
\text { protección }\end{array}$ & 3 & 42.9 & 119 & 100.0 & 5 & 71.4 \\
$\begin{array}{l}\text { Historial de enfermedad por } \\
\text { brucelosis anterior }\end{array}$ & 0 & 0 & 118 & 99.2 & 3 & 42.9 \\
$\begin{array}{l}\text { Historial de enfermedad por } \\
\text { brucelosis de los familiares }\end{array}$ & 0 & 0 & 0 & 0 & 1 & 14.3 \\
$\begin{array}{l}\text { No investigación de brucelosis en } \\
\text { las personas }\end{array}$ & 0 & 0 & 0 & 0 & 0 & 0.00 \\
\hline Promedio & 6 & 85.7 & 119 & 100.0 & 7 & 100.0 \\
\hline
\end{tabular}

En general, se pudo observar que las mayores frecuencias de FRP se presentaron en las lecherías procesadoras de leche (79.2\%), seguido por los mataderos $(51.4 \%)$, y en último lugar se encuentran los centros de procesamiento de leche $(30.4 \%)(\mathrm{p}<0.05)$. Asimismo, se determinó que 3 de 7 centros de procesamiento de leche $(57.1 \%)$ y 19 de 119 lecherías procesadoras de leche $(16.0 \%)$ resultaron positivos a la prueba del anillo de leche $(\mathrm{p}<0.05)$.

\section{Discusión}

Las deficientes condiciones constructivas, la mala higiene y bioseguridad de las instalaciones de los centros que se dedican a procesar alimentos, pueden constituir un serio riesgo para contaminación de estos por agentes patológicos y presentar un riesgo ocupacional para los trabajadores que laboran en los mismos (Cook et al., 2017).
Como resultado de la encuesta se pudo comprobar la existencia de serias deficiencias en la higiene y bioseguridad de los centros en estudio, especialmente en las lecherías procesadoras de leche y en los mataderos. La presencia de otras especies de animales, la deficiente higiene de las instalaciones y utensilios, la ausencia de desinfecciones, de filtro sanitario y de tratamiento de residuales se observó con frecuencia. Está ampliamente descrito (Lytras et al., 2016) que la brucelosis en los humanos es una enfermedad ocupacional, ya que el contacto directo con los animales y ambientes contaminados constituye el mecanismo fundamental de transmisión de la enfermedad (Alim et al., 2015), de ahí que la higiene y la bioseguridad juegan un papel primordial en su control (Cowie et al., 2014).

En el análisis de los factores de riesgo asociados al personal que labora en los centros procesadores de alimentos se pudo 
comprobar que todos los encuestados refieren que consumen alimentos considerados como riesgosos y que no se utilizan adecuadamente los medios de protección, sobre todo en los mataderos y en las lecherías procesadoras de leche. Se tiene evidencia de la ocurrencia de brucelosis en personas en países asiáticos por la ingestión de fetos (Yoo et al., 2015) y por la ingestión de leche sin tratamiento previo en Gambia y Senegal (Bankole et al., 2011). Asimismo, Nabukenya et al. (2013) reportó como factor de riesgo de importancia en mataderos la carencia de ropa de protección.

El queso fresco constituyó el principal producto elaborado a partir de la leche, el cual no se pasteuriza en el $42 \%$ de los centros de procesamiento de leche ni en el $100 \%$ de las lecherías procesadoras de leche. Situaciones similares han sido detectadas en productores de quesos frescos en la zona central de México (García-Juárez et al., 2014). Un problema adicional es que estos centros no efectúan la prueba de anillo de la leche y, por lo tanto, no pueden detectar si la leche acopiada procede de hatos afectados por la brucelosis. En este estudio se pudo comprobar que muestras de leche de 4 de los 7 centros de procesamiento de leche resultaron positivas a esta prueba.

Esta situación representa un serio riesgo para la salud pública, ya que el consumo de leche y quesos sin pasteurizar, procedentes de rebaños afectados por brucelosis, constituye uno de los mecanismos fundamentales de transmisión de la enfermedad a los humanos (Atluri et al., 2011; Megersa et al., 2011; Yumuka y O'Callaghan, 2012). En algunos países de África, más del $60 \%$ de los encuestados respondieron consumir leche sin previo tratamiento (Bankole et al., 2011; Tebug et al., 2015), mientras que, en Uganda, Asiimwe et al. (2015) señalan al consumo de mantequilla y leche sin pasteurizar como factores de riesgo. Asimismo, en un estudio realizado en pacientes afectados por brucelosis, se demostró que el 91\% presentaba un historial de consumo de queso fresco (Alim et al., 2015).

Un aspecto primordial para el control de la brucelosis en personas que se encuentra en el grupo de alto riesgo de padecer la enfermedad es la evaluación serológica periódica (Mangalgi et al., 2016). Sin embargo, en este estudio se comprobó que no se investiga periódicamente al personal que labora en estos centros procesadores; por tanto, se incumple lo establecido en las buenas prácticas ganaderas con relación a la protección del personal (FAO y FIL, 2012).

\section{Conclusiones}

- Se observa un elevado porcentaje de factores de riesgo para la transmisión de brucelosis al personal en lecherías que procesan leche, en centros procesadores de leche y en mataderos, especialmente en el primer caso.

- La prueba del anillo resultó positiva a Brucella spp en el 57.1\% (4/7) de muestras de leche de centros procesadores de leche y en el 16.0\% (19/119) de lecherías que procesan leche.

\section{Literatura Citada}

1. AGROCALIDAD. 2009. Programa nacional de control de brucelosis bovina. Resolución Sanitaria N. ${ }^{\circ} 025$. Ecuador. [Internet]. Disponible en: http:// www.agrocalidad.gob.ec/agrocalidad/ images/pdfs/sanidadanimal/ programa_nacional_brucelosis_bovina.pdf

2. Alim A, Oguzkaya-Artan M, Artan C. 2015. The seroprevalence of brucellosis among undiagnosed family members of brucellosis positive patients. Niger J Clin Pract 18: 620-625. doi: 10.4103/11193077.154206 
3. Aréstegui M, Gualtieri C, Domínguez J, Scharovsky G. 2001. El género Brucella y su interacción con el sistema mononuclear fagocítico. Vet Mex 32: 131-139.

4. Asiimwe BB, Kansiime C, Rwego IB. 2015. Risk factors for human brucellosis in agro-pastoralist communities of south western Uganda: a case-control study. BMC Res Notes 8: 405. doi: 10.1186/ s13104-015-1361-z

5. Atluri V, Xavier M, de Jong MD, den Hartigh AB, Tsolis R. 2011. Interactions of the human pathogenic Brucella species with their hosts. Annu Rev Microbiol 65: 523-541. doi: 10.1146/ annurev-micro-090110-102905

6. Bankole AA, Secka A, Ly C. 2011. Risk behaviours for milk-borne diseases transmission along the milk chain in The Gambia and Senegal. Trop Anim Health Prod 43: 103-109. doi: 10.1007/s11250010-9660-9

7. Bhanu RV, Gunaseelan L, Subramanian A, Yale G 2013. A study on bovine brucellosis in an organized dairy farm. Vet World 6: 681-685. doi: 10.14202/vetworld.2013.681-685

8. Cook EAJ, de Glanville WA, Thomas LF, Kariuki S, de Clare Bronsvoort, BM, Fèvre EM. 2017. Working conditions and public health risks in slaughterhouses in western Kenya. BMC Public Health 17: 14. doi: 10.1186/ s12889-016-3923-y

9. Cowie CE, Marreos N, Gortázar C, Jaroso R, White PCL, Balseiro A. 2014. Shared risk factors for multiple livestock diseases: a case study of bovine tuberculosis and brucellosis. Res Vet Sci 97:491-497. doi: 10.1016/j.rvsc.2014.09.002

10. D'Anastasio R, Staniscia MT, Milia ML, Manzoli L, Capasso L. 2011. Origin, evolution and paleoepidemiology of brucellosis. Epidemiol Infect 139: 149156. doi: 10.1017/S095026881000097X
11. Dean AS, Crump L, Greter $H$, Schelling E, Zinsstag Z. 2012. Global burden of human brucellosis: a systematic review of disease frequency. PLoS Negl Trop Dis 6: e1865. doi: 10.1371/ journal.pntd.0001865

12. [FAO] Organización de las Naciones Unidas para la Alimentación y la Agricultura, [FIL] Federación Internacional de la Leche. 2012. Guía de buenas prácticas en explotaciones lecheras. Directrices FAO: Producción y Sanidad Animal N. ${ }^{\circ}$ 8. Roma. [Internet]. Disponible en: http://www.fao.org/ docrep/015/ba0027s/ba0027s00.pdf

13. García-Juárez G, Ramírez-Bribiesca JE, Hernández-Vázquez M, Hernández-Calva LM, Díaz-Aparicio E, Orozco-Bolaños H. 2014. Análisis de riesgos de la brucelosis en el estado de Tlaxcala. Salud Pública Méx 56: 355-362.

14. Lytras T, Danis K, Dounias G 2016. Incidence patterns and occupational risk factors of human brucellosis in Greece, 2004-2015. Int J Occup Environ Med 7: 221-226.

15. Luigi PF, Mastrandrea S, Rappelli P, Cappuccinelli P. 2006. Brucella abortus infection acquired in microbiology laboratories. J Clin Microbiol 38: 2000-2005

16. Mangalgi SS, Sajjan AG, Mohite ST, Gajul S. 2016. Brucellosis in occupationally exposed groups. J Clin Diagn Res 4: DC24-DC27. doi: 10.7860/ JCDR/2016/15276.7673

17. Megersa B, Biffa D, Niguse F, Rufael T, Asmare K, Skjerve E. 2011. Cattle brucellosis in traditional livestock husbandry practice in Southern and Eastern Ethiopia, and its zoonotic implication. ActaVet Scand 53: 24. doi: 10.1186/1751-0147-53-24

18. Motarjemi Y, Moy GG, Jooste PJ, Anelich LE. 2014. Milk and dairy products. In: Motarjemi Y, Lelieveld H (eds). Food safety and management. A practical guide for the food industry. USA: Elsevier. p 83-117. 
19. Nabukenya I, Kaddu-Mulindwa D, Nasinyama GW. 2013. Survey of Brucella infection and malaria among abattoir workers in Kampala and Mbarara districts, Uganda. BMC Public Health 13: 901 .doi:10.1186/1471-2458-13-901

20. [OIE] Organización Mundial de Sanidad Animal. 2016. Brucelosis (Brucella abortus, Brucella melitensis y Brucella suis). En: Manual de las pruebas de diagnóstico y de las vacunas para los animales terrestres. $7^{\mathrm{a}} \mathrm{ed}$. Vol 1, Cap 2.1.4. Paris: OIE. [Internet]. Disponible en: http://www.oie.int/fileadmin/Home/ esp/Health_standards/tahm/ 2.01.04_BOVINE_ERUCELL.pdf

21. [OPS] Organización Panamericana de la Salud, [OMS] Organización Mundial de la Salud. 2005. 14 ${ }^{\mathrm{a}} \mathrm{Re}-$ unión Interamericana a Nivel Ministerial en Salud y Agricultura. Ciudad de México, México. [Internet]. Disponible en: http://bvs.panalimentos.org/local/File/ COPAIA4-inf-final.pdf

22. Osman AEF, Hassan AN, Ali AE, Abdoel TH, Smits HL. 2015. Brucella melitensis biovar 1 and Brucella abortus $\mathrm{S} 19$ vaccine strain infections in milkers working at cattle farms in the Khartoum Area, Sudan. PLoS ONE 10: e0123374. doi: 10.1371/journal.pone.0123374

23. Paredes-Vargas SR. 2012. Determinar la prevalencia de brucelosis bovina y factores de riesgo en la parroquia Alluriquin, recinto Cristal de Lelia. Tesis de Ingeniero Agropecuario. Santo Domingo de los Tsáchilas, Ecuador: Escuela Politécnica del Ejército. 129 p.
24. Ron-Román J, Ron-Garrido L, Abatih E, Celi-Erazo M, Vizcaíno-Ordóñez L, Calva-Pacheco J, González-Andrade $P$, et al. 2014. Human brucellosis in North-West Ecuador: typifying Brucella spp, sero-1 prevalence, and associated risk factors. Vector Borne Zoonotic Dis14: 124-133. doi: 10.1089/ vbz.2012.1191

25. Tebug SF, Kamga-Waladjo AR, Ema PJ, Muyeneza C, Kane O, Seck A, Ly MT, Lo M. 2015. Cattle farmer awareness and behavior regarding prevention of zoonotic disease transmission in Senegal. J Agromedicine 20: 217-224. doi: 10.1080/1059924X.2015.1010068

26. Yoo JR, Heo ST, Lee KH, Kim YR, Yoo SR. 2015. Foodborne outbreak of human brucellosis caused by ingested raw materials of fetal calf on Jeju Island. Am J Trop Med Hyg 92: 267-269. doi: 10.4269/ajtmh.14-0399

27. Yumuka Z, O'Callaghan D. 2012. Brucellosis in Turkey - an overview. Int J Infect Dis 16: e228-e235. doi: 10.1016/ j.ijid.2011.12.011

28. Zambrano Aguayo MD, Pérez Ruano M. 2015. Seroprevalencia de brucelosis en ganado bovino y en humanos vinculados a la ganadería bovina en las zonas norte y centro de la provincia Manabí, Ecuador. Rev Salud Anim 37: 164-172.

29. Zhong Z, Yu S, Wang X, Dong S, Xu $J$, Wang $Y$, Chen $Z$, Ren Z, Peng $G$. 2013. Human brucellosis in the People's Republic of China during 2005-2010. Int J Infect Dis 17: e289-e292. doi: 10.1016/ j.ijid.2012.12.030 